Supporting Information for

\title{
Versatile, Fragrant, Convertible Isonitriles
}

\author{
Michael C. Pirrung* and Subir Ghorai \\ Department of Chemistry, University of California, Riverside, CA 92521 \\ michael.pirrung@ucr.edu
}

General Information. Organic solvents were concentrated under reduced pressure on a Büchi rotary evaporator. Chromatographic purification of products was accomplished using flash column chromatography on silica gels. Thin-layer chromatography (TLC) was carried out on aluminium sheets, Silica Gel $60 \mathrm{~F}_{254}$ (Merck; layer thickness $0.25 \mathrm{~mm}$ ). Visualization of the developed chromatogram was performed by UV light and $\mathrm{KMnO}_{4}$ stain. Solutions were dried over anhydrous $\mathrm{Na}_{2} \mathrm{SO}_{4}$.

All melting points were measured on a Büchi Melting Point B-545 and are uncorrected. ${ }^{1} \mathrm{H}$ and ${ }^{13} \mathrm{C}$ NMR spectra were recorded on Varian Inova 300 (300 MHz and $75 \mathrm{MHz}$ respectively) as noted, and are internally referenced to residual protio solvent signals. Data for ${ }^{1} \mathrm{H}$ are reported as follows: chemical shift $(\delta \mathrm{ppm})$, multiplicity (s = singlet, $\mathrm{d}=$ doublet, $\mathrm{t}=$ triplet, $\mathrm{q}=$ quartet, $\mathrm{m}=$ multiplet, sep = septet), coupling constant and integration. Data for ${ }^{13} \mathrm{C}$ NMR are reported in terms of chemical shift. IR spectra were recorded on a Perkin Elmer Spectrum One FT-IR spectrometer using the ATR accessory and are reported in absorption frequency $\left(\mathrm{cm}^{-1}\right)$. Mass spectra were obtained from the University of California, Riverside Mass Spectral facility.

General Procedure for Isonitrile Formation: A $50 \mathrm{~mL}$ round-bottom flask equipped with a magnetic stir bar and charged with benzoxazole (0.90 g, $7.56 \mathrm{mmol}$ ) and THF (18 mL) are allowed to cool to $-78{ }^{\circ} \mathrm{C}$ for five min prior to addition of $n$-BuLi (1.6 M solution in hexanes, $4.96 \mathrm{~mL}, 7.94 \mathrm{mmol}$ ). The reaction mixture was allowed to stir at the same temperature for $1.5 \mathrm{~h}$. The acid chloride $(7.94 \mathrm{mmol})$ was added dropwise to the solution. The solution was allowed to warm to room temperature and stirred for $2 \mathrm{~h}$. The reaction mixture was poured onto a mixture of ether $(100 \mathrm{~mL})$ and saturated aqueous 
$\mathrm{NaHCO}_{3}(50 \mathrm{~mL})$. The organic layer was washed with water $(2 \times 50 \mathrm{~mL})$, dried, and concentrated in vacuo. The resulting residue was purified by silica gel flash column chromatography (solvent noted) and the organics concentrated in vacuo to provide the title compounds.<smiles>CC1=[Nb]C=CO1</smiles>

2-isocyanovinyl acetate (8a): Eluting with hexanes to 99:1 hexanes/ethyl acetate gradient to provide the title compound as a brown liquid (75\%). ${ }^{1} \mathrm{H}$ NMR (300 MHz, $\mathrm{CDCl}_{3}$ ): $\delta 7.38$ (br d, $J=4.2 \mathrm{~Hz}, 1 \mathrm{H}$ ), 5.38 (d, $J=4.8 \mathrm{~Hz}, 1 \mathrm{H}$ ), 2.28 (s, 3H); ${ }^{13} \mathrm{C}$ NMR (75 MHz, $\mathrm{CDCl}_{3}$ ): $\delta 169.8,166.3,138.3,97.5,20.4$; IR (film): 3108, 2128, 1771, 1660, 1432, 1375, 1364, 1181, 1099, 1045, $1007 \mathrm{~cm}^{-1}$; MS (CI): m/z 112 (M + H).<smiles>COC(=O)C(C(=O)OC)C(c1ccccc1)c1ccccc1</smiles>

(R)-2-isocyanovinyl 2-(formyloxy)-2-phenylacetate (8b): Pale yellow liquid (95\%). ${ }^{1} \mathrm{H}$ NMR (300 MHz, CDCl $): \delta 8.23$ (s, 1H), 7.56-7.33 (m, 6H), 6.18 (s, 1H), 5.43 (d, $J=4.5$ Hz, 1H); IR (film): 3109, 2958, 2126, 1782, 1726, 1661, 1497, 1456, 1334, 1232, 1197, 1132, 1090, $1003 \mathrm{~cm}^{-1}$; MS (CI): m/z $232(\mathrm{M}+\mathrm{H})$.<smiles>CC(=O)Oc1ccccc1N</smiles>

2-isocyanophenyl acetate (10a): Eluting with hexanes to 49:1 hexanes/ethyl acetate gradient to provide the title compound as a liquid (85\%). ${ }^{1} \mathrm{H}$ NMR (300 MHz, $\mathrm{CDCl}_{3}$ ): $\delta$ 7.41-7.35 (m, 2H), 7.24-7.17 (m, 2H), 2.35 (s, 3H); ${ }^{13} \mathrm{C} \mathrm{NMR} \mathrm{(75} \mathrm{MHz,} \mathrm{CDCl}_{3}$ ): $\delta 169.5$, 168.2, 146.6, 130.6, 127.7, 126.8, 123.7, 120.5, 20.9; IR (film): 2125, 1772, 1490, 1455, 1370, 1238, 1191, 1167, 1101, 1033, $1009 \mathrm{~cm}^{-1}$; MS (CI): m/z $179\left(\mathrm{M}+\mathrm{NH}_{4}\right), 162(\mathrm{M}+$ $\mathrm{H})$. 
<smiles>CC(C)(C)C(=O)Oc1ccccc1N</smiles>

2-isocyanophenyl pivalate (10b): Eluting with hexanes to 39:1 hexanes/ethyl acetate gradient to provide the title compound as a liquid (92\%). ${ }^{1} \mathrm{H}$ NMR (300 $\mathrm{MHz}, \mathrm{CDCl}_{3}$ ): $\delta$ 7.43-7.38 (m, 2H), 7.27-7.16 (m, 2H), 1.42 (s, 9H); ${ }^{13} \mathrm{C}$ NMR (75 MHz, $\mathrm{CDCl}_{3}$ ): $\delta$ 176.0, 169.3, 146.8, 130.5, 127.8, 126.6, 123.7, 120.6, 39.4, 27.4; IR (film): 2977, 2875, 2125, 1762, 1491, 1480, 1399, 1368, 1270, 1235, 1088, $1027 \mathrm{~cm}^{-1}$; MS (CI): m/z 221 (M + $\left.\mathrm{NH}_{4}\right)$.<smiles></smiles>

2-isocyanophenyl 4-methylbenzoate (10c): Eluting with hexanes to 32:1 hexanes/ethyl acetate gradient to provide the title compound as a white solid (96\%). mp: 114.2-114.9 ${ }^{\circ} \mathrm{C} ;{ }^{1} \mathrm{H}$ NMR (300 MHz, $\left.\mathrm{CDCl}_{3}\right): \delta 8.15$ (d, $\left.J=8.1 \mathrm{~Hz}, 2 \mathrm{H}\right), 7.44$ (td, $J=7.8,1.8 \mathrm{~Hz}$, 2H), 7.39 (dd, $J=7.2,1.8 \mathrm{~Hz}, 1 \mathrm{H}$ ), 7.33 (d, $J=8.1 \mathrm{~Hz}, 2 \mathrm{H}$ ), 7.29 (dd, $J=7.8,1.8 \mathrm{~Hz}$, 1H), 2.45 (s, 3H); ${ }^{13} \mathrm{C}$ NMR (75 MHz, $\left.\mathrm{CDCl}_{3}\right): \delta$ 169.6, 164.2, 146.9, 145.4, 130.7, 130. 6, 129.8, 127.8, 126.7, 125.9, 123.9, 120.6, 22.1; IR (film): 3064, 2126, 1734, 1612, 1509, 1489, 1447, 1265, 1234, 1172, 1102, 1064, $1020 \mathrm{~cm}^{-1}$; MS (CI): m/z 255 (M + $\left.\mathrm{NH}_{4}\right), 119$.<smiles>CC(C)(C)OC(=O)Oc1ccccc1N</smiles>

tert-Butyl 2-isocyanophenyl carbonate (10d): Eluting with hexanes to 39:1 hexanes/ethyl acetate gradient to provide the title compound as a liquid (83\%). ${ }^{1} \mathrm{H}$ NMR (300 MHz, $\mathrm{CDCl}_{3}$ ): $\delta$ 7.41-7.35 (m, 2H), 7.25-7.19 (m, 2H), 1.55 (s, 9H); ${ }^{13} \mathrm{C}$ NMR (75 $\mathrm{MHz}_{\mathrm{CDCl}}$ ): $\delta 170.0,150.6,146.8,130.6,127.8,126.9,123.4,120.7,85.2$, 27.8; IR (film): 2984, 2124, 1762, 1492, 1397, 1372, 1283, 1260, 1231, 1139, 1102, 1046, 1007 $\mathrm{cm}^{-1}$; MS (CI): m/z $237\left(\mathrm{M}+\mathrm{NH}_{4}\right), 220(\mathrm{M}+\mathrm{H})$. 
<smiles>COc1ccc(C(=O)Oc2ccccc2N)cc1</smiles>

2-isocyanophenyl 4-methoxybenzoate (10e): Eluting with hexanes to 9:1 hexanes/ethyl acetate gradient to provide the title compound as a white solid (96\%). mp: 108.5-110.5 ${ }^{\circ} \mathrm{C} ;{ }^{1} \mathrm{H}$ NMR (300 MHz, $\mathrm{CDCl}_{3}$ ): $\delta 8.18$ (d, $\left.J=8.7 \mathrm{~Hz}, 2 \mathrm{H}\right), 7.45-7.34$ (m, 3H), 7.24 (t, $J$ $=7.5 \mathrm{~Hz}, 1 \mathrm{H}), 6.98$ (d, $J=8.7 \mathrm{~Hz}, 2 \mathrm{H}), 3.85$ (s, 3H); ${ }^{13} \mathrm{C}$ NMR $\left(75 \mathrm{MHz}, \mathrm{CDCl}_{3}\right): \delta$ 169.4, 164.6, 163.8, 147.0, 132.9, 130.5, 127.8, 126.6, 123.9, 120.9, 120.6, 114.3, 55.8; IR (film): 2958, 2845, 2128, 1729, 1606, 1581, 1509, 1489, 1423, 1279, 1256, 1233, $1168 \mathrm{~cm}^{-1}$; MS (CI): m/z $271\left(\mathrm{M}+\mathrm{NH}_{4}\right), 254(\mathrm{M}+\mathrm{H}), 135$.<smiles>N#Cc1ccc(C(=O)Oc2ccccc2N)cc1</smiles>

2-isocyanophenyl 4-cyanobenzoate (10f): Eluting with hexanes to 12:1 hexanes/ethyl acetate gradient to provide the title compound as a white solid (90\%). mp: 159.7-161.3 ${ }^{\circ} \mathrm{C} ;{ }^{1} \mathrm{H}$ NMR (300 MHz, $\mathrm{CDCl}_{3}$ ): $\delta 8.35$ (d, $\left.J=8.1 \mathrm{~Hz}, 2 \mathrm{H}\right), 7.84$ (d, $\left.J=8.1 \mathrm{~Hz}, 2 \mathrm{H}\right)$, 7.52-7.48 (m, 2H), 7.42-7.32 (m, 2H); ${ }^{13} \mathrm{C} \mathrm{NMR} \mathrm{(75} \mathrm{MHz,} \mathrm{CDCl}_{3}$ ): $\delta$ 169.9, 162.6, 146.3, 132.8, 132.4, 131.1, 130.8, 128.0, 127.3, 123.5, 120.3, 117.9, 117.8; IR (film): 3050, 2239, 2128, 1738, 1610, 1488, 1448, 1410, 1296, 1266, 1231, 1179, 1104, 1076 $\mathrm{cm}^{-1}$; MS (CI): m/z $266\left(\mathrm{M}+\mathrm{NH}_{4}\right), 249(\mathrm{M}+\mathrm{H}), 130$.<smiles>Nc1ccccc1OC(=O)c1ccccc1</smiles>

2-isocyanophenyl benzoate (10g): Eluting with hexanes to 12:1 hexanes/ethyl acetate gradient to provide the title compound as a white solid (93\%). mp: 91.8-92.7 ${ }^{\circ} \mathrm{C} ;{ }^{1} \mathrm{H}$ NMR (300 MHz, $\left.\mathrm{CDCl}_{3}\right): \delta 8.26$ (dd, $\left.J=8.1,1.2 \mathrm{~Hz}, 2 \mathrm{H}\right), 7.64(\mathrm{td}, J=8.4,1.2 \mathrm{~Hz}, 1 \mathrm{H})$, 7.51 (t, $J=7.8 \mathrm{~Hz}, 2 \mathrm{H}), 7.45-7.35$ (m, 3H), 7.24 (td, $J=8.4,1.8 \mathrm{~Hz}, 1 \mathrm{H}) ;{ }^{13} \mathrm{C}$ NMR (75 $\left.\mathrm{MHz}_{\mathrm{CDCl}}\right): \delta$ 169.8, 164.1, 146.8, 134.5, 130.7, 129.1, 128.7, 127.8, 126.9, 123.9, 
120.5; IR (film): 3047, 2131, 1732, 1601, 1586, 1492, 1449, 1257, 1231, 1172, 1098, 1078, 1061, 1037, 1023, $1001 \mathrm{~cm}^{-1}$; MS (CI): m/z $241\left(\mathrm{M}+\mathrm{NH}_{4}\right), 224(\mathrm{M}+\mathrm{H})$.

General Procedure for Ugi Reaction: To a solution of amine $(1.0 \mathrm{mmol})$ in methanol $(2.0 \mathrm{~mL})$ was added aldehyde $(1.0 \mathrm{mmol})$, and the reaction mixture was stirred at room temperature for $10 \mathrm{~min}$. To this solution was added carboxylic acid (1.0 mmol), and the reaction mixture was stirred for $5 \mathrm{~min}$, then isonitrile $(1.0 \mathrm{mmol})$ was added. The resulting mixture was stirred at room temperature until the reaction was determined to be complete by TLC. The resulting solution was concentrated in vacuo and purified by silica gel flash column chromatography (solvents noted) to provide the title compounds.<smiles>CCC(=O)N(Cc1ccccc1)C(C(=O)N/C=C\OC(C)=O)C(C)C</smiles>

(Z)-2-(2-(N-benzylpropionamido)-3-methylbutanamido) vinyl acetate (14): Prepared according to the general procedure from $\mathbf{8 a}$ for $12 \mathrm{~h}$ to provide title compound as a white solid (74\%) after purification by flash column chromatography on silica gel, eluting with hexanes to 10:1 hexanes/ethyl acetate gradient. mp: 84.5-87.6 ${ }^{\circ} \mathrm{C}$; ${ }^{1} \mathrm{H}$ NMR (300 MHz, $\mathrm{CDCl}_{3}$ ): $\delta 8.75$ (br s, 1H), 7.33-7.22 (m, 3H), 7.09 (d, $\left.J=6.9 \mathrm{~Hz}, 2 \mathrm{H}\right), 6.75$ (d, $J=4.8$ Hz, 1H), 6.34 (dd, $J=11.1,5.4 \mathrm{~Hz}, 1 \mathrm{H}$ ), 4.64 (d, $J=17.1 \mathrm{~Hz}, 1 \mathrm{H}$ ), 4.56 (d, $J=17.1 \mathrm{~Hz}$, 1H), 4.28 (d, $J=10.5 \mathrm{~Hz}, 1 \mathrm{H}$ ), 2.63-2.55 (m, 1H), 2.48-2.29 (m, 2H), 2.25 (s, 3H), 1.12 (t, $J=7.5 \mathrm{~Hz}, 3 \mathrm{H}), 0.95$ (d, $J=6.6 \mathrm{~Hz}, 3 \mathrm{H}), 0.85$ (d, $J=6.9 \mathrm{~Hz}, 3 \mathrm{H}) ;{ }^{13} \mathrm{C}$ NMR $(75 \mathrm{MHz}$, $\left.\mathrm{CDCl}_{3}\right): \delta 176.9,167.7,167.1,136.9,128.9,127.7,126.5,121.4,108.5,67.5,50.6,27.7$, 26.9, 20.8, 20.0, 19.3, 9.7; IR (film): 3324, 2960, 2938, 1754, 1693, 1682, 1631, 1517, 1497, 1452, 1417, 1369, 1355, 1201, 1175, 1145, $1116 \mathrm{~cm}^{-1}$; MS (FAB): $\mathrm{m} / \mathrm{z} 347$ (M + H), 246. 
<smiles>CCC(=O)N(Cc1ccccc1)C(C(=O)Nc1ccccc1OC(=O)c1ccc(C)cc1)C(C)C</smiles>

2-(2-( $N$-benzylpropionamido)-3-methylbutanamido)phenyl 4-methylbenzoate (16):

Prepared according to the general procedure from 10c for $12 \mathrm{~h}$ to provide title compound as a pale yellow liquid (90\%) after purification by flash column chromatography on silica gel, eluting with $\mathrm{CH}_{2} \mathrm{Cl}_{2}$ to $13: 1 \mathrm{CH}_{2} \mathrm{Cl}_{2}$ /methanol gradient. ${ }^{1} \mathrm{H} \mathrm{NMR}$ (300 $\mathrm{MHz}, \mathrm{CDCl}_{3}$ ): $\delta 9.08$ (br s, 1H), 8.19 (d, $J=8.4 \mathrm{~Hz}, 2 \mathrm{H}), 8.13$ (d, $J=7.5 \mathrm{~Hz}, 1 \mathrm{H}), 7.33$ (d, $J=8.4 \mathrm{~Hz}$, 2H), 7.21-7.05 (m, 8H), 4.55 (s, 2H), 4.36 (br d, $J=9.9$ Hz, 1H), 2.61-2.49 (m, 1H), 2.46 (s, 3H), 2.25-2.06 (m, 2H), 0.97 (d, $J=6.3 \mathrm{~Hz}, 3 \mathrm{H}), 0.81$ (t, $J=8.4 \mathrm{~Hz}, 3 \mathrm{H}), 0.80$ (d, $J=$ $6.3 \mathrm{~Hz}, 3 \mathrm{H}) ;{ }^{13} \mathrm{C}$ NMR (75 MHz, $\left.\mathrm{CDCl}_{3}\right): \delta$ 176.9, 169.2, 165.1, 144.9, 140.8, 136.9, 131.0, 130.9, 129.6, 129.0, 127.6, 126.6, 126.5, 124.5, 122.7, 122.3, 68.2, 50.4, 43.9, 27.5, 26.5, 22.0, 20.3, 19.4, 9.3; IR (film): 3272, 2966, 2874, 1738, 1687, 1634, 1608, 1528, 1452, 1413, 1264, 1240, 1173, 1104, 1058, $1018 \mathrm{~cm}^{-1}$; MS (CI): m/z 473 (M+ H).<smiles>COc1ccc(CN(C(C)=O)C(C(=O)Nc2ccccc2OC(=O)c2ccc(C)cc2)c2ccccc2)cc1</smiles>

2-(2-(N-(4-methoxybenzyl)acetamido)-2-phenylacetamido)phenyl 4-methylbenzoate (18): Prepared according to the general procedure from 10c for $24 \mathrm{~h}$ to provide title compound as a colorless liquid (60\%) after purification by flash column chromatography on silica gel, eluting with hexanes to $1: 1$ hexanes/ethyl acetate gradient. ${ }^{1} \mathrm{H}$ NMR (300 $\mathrm{MHz} \mathrm{CDCl}_{3}$ ): $\delta 8.18$ (d, $\left.J=7.5 \mathrm{~Hz}, 1 \mathrm{H}\right), 8.01$ (s, 1H), 7.81 (d, $\left.J=7.5 \mathrm{~Hz}, 2 \mathrm{H}\right), 7.24-$ 7.17 (m, 5H), 7.13-7.09 (m, 2H), 7.06-7.01 (m, 3H), 6.89 (d, $J=8.1 \mathrm{~Hz}, 2 \mathrm{H}), 6.69$ (d, $J=$ 
$8.1 \mathrm{~Hz}, 2 \mathrm{H}$ ), 5.79 (s, 1H), 4.59 (d, $J=17.1 \mathrm{~Hz}, 1 \mathrm{H}$ ), 4.37 (d, $J=17.1 \mathrm{~Hz}, 1 \mathrm{H}$ ), 3.68 (s, 3H), 2.42 (s, 3H), 2.00 (s, 3H); ${ }^{13} \mathrm{C} \mathrm{NMR} \mathrm{(75} \mathrm{MHz,} \mathrm{CDCl}_{3}$ ): $\delta$ 172.6, 168.1, 164.6, 159.0, 144.9, 141.3, 134.8, 130.6, 130.3, 129.7, 129.5, 129.0, 128.8, 127.8, 126.6, 126.1, 125.0, 123.1, 122.5, 114.2, 64.8, 55.4, 53.8, 51.3, 22.5, 22.0; IR (film): 3267, 3033, 1737, 1698, 1631, 1609, 1512, 1452, 1408, 1242, 1173, 1105, $1059 \mathrm{~cm}^{-1}$; MS (FAB): $\mathrm{m} / \mathrm{z} 523$ (M + $\mathrm{H})$.<smiles>COc1ccc(C(=O)Oc2ccccc2NC(=O)C(C(C)C)N(C(C)=O)C2CC2)cc1</smiles>

\section{2-(2-(N-cyclopropylacetamido)-3-methylbutanamido)phenyl 4-methoxybenzoate}

(22e): Prepared according to the general procedure from 10e for $15 \mathrm{~h}$ to provide title compound as a pale orange solid (90\%) after purification by flash column chromatography on silica gel, eluting with hexanes to 3:1 hexanes/ethyl acetate gradient. mp: 106.1-107.5 ${ }^{\circ} \mathrm{C} ;{ }^{1} \mathrm{H}$ NMR (300 MHz, $\mathrm{CDCl}_{3}$ ): $\delta 9.62$ (br s, $1 \mathrm{H}$ ), 8.26 (d, $J=8.1 \mathrm{~Hz}$, 1H), 8.22 (d, $J=8.7 \mathrm{~Hz}, 2 \mathrm{H}$ ), 7.23 (dd, $J=8.1,4.5 \mathrm{~Hz}, 1 \mathrm{H}$ ), 7.12-7.10 (m, 2H), 7.01 (d, $J$ $=8.7 \mathrm{~Hz}, 2 \mathrm{H}), 3.95-3.91(\mathrm{~m}, 4 \mathrm{H}), 2.78-2.70(\mathrm{~m}, 1 \mathrm{H}), 2.66-2.59(\mathrm{~m}, 1 \mathrm{H}), 1.87(\mathrm{~s}, 3 \mathrm{H})$, 1.03 (d, $J=6.3 \mathrm{~Hz}, 3 \mathrm{H}$ ), 1.02-0.94 (m, 1H), 0.87 (d, $J=6.3 \mathrm{~Hz}, 3 \mathrm{H}$ ), 0.86-0.73 (m, 3H); ${ }^{13} \mathrm{C}$ NMR (75 MHz, $\left.\mathrm{CDCl}_{3}\right): \delta 175.7,170.4,164.6,164.2,141.1,132.9,131.4,126.5$, 124.5, 122.6, 121.7, 114.0, 72.5, 55.7, 32.7, 26.6, 22.9, 20.2, 9.6, 9.1; IR (film): 3236, 2968, 1732, 1683, 1634, 1605, 1533, 1511, 1453, 1302, 1243, 1163, 1102, 1056, 1025, $1006 \mathrm{~cm}^{-1}$; MS (FAB): m/z $425(\mathrm{M}+\mathrm{H}), 182$.<smiles>CC(=O)N(C1CC1)C(C(=O)Nc1ccccc1OC(=O)c1ccc(C#N)cc1)C(C)C</smiles> 
2-(2-( $N$-cyclopropylacetamido)-3-methylbutanamido)phenyl 4-cyanobenzoate (22f): Prepared according to the general procedure from $10 \mathrm{f}$ for $15 \mathrm{~h}$ to provide title compound as a white solid (87\%) after purification by flash column chromatography on silica gel, eluting with hexanes to 4:1 hexanes/ethyl acetate gradient. mp: 117.4-118.6 ${ }^{\circ} \mathrm{C} ;{ }^{1} \mathrm{H}$ NMR (300 MHz, CDCl $): \delta 9.85$ (br s, 1H), 8.36 (d, $J=8.1 \mathrm{~Hz}, 2 \mathrm{H}$ ), 8.29 (d, $J=8.4 \mathrm{~Hz}, 1 \mathrm{H}$ ), 7.81 (d, $J=7.8 \mathrm{~Hz}, 2 \mathrm{H}), 7.27-7.21$ (m, $1 \mathrm{H}), 7.12-7.09$ (m, 2H), 3.81 (d, $J=10.5 \mathrm{~Hz}, 1 \mathrm{H}$ ), 2.74-2.62 (m, 2H), 1.88 (s, 3H), 0.98 (d, $J=6.3 \mathrm{~Hz}, 3 \mathrm{H}$ ), 0.85 (d, $J=6.3 \mathrm{~Hz}, 3 \mathrm{H}), 0.99-$ 0.71 (m, 4H); ${ }^{13} \mathrm{C}$ NMR (75 MHz, $\mathrm{CDCl}_{3}$ ): $\delta$ 175.8, 170.3, 163.5, 140.2, 133.5, 132.4, 131.3, 131.2, 127.1, 124.4, 122.4, 122.3, 118.2, 117.1, 73.6, 33.2, 26.6, 23.1, 20.2, 20.1, 10.0, 9.1; IR (film): 3256, 2966, 2873, 2232, 1740, 1691, 1674, 1628, 1608, 1528, 1454, 1390, 1304, 1252, 1174, 1107, $1069 \mathrm{~cm}^{-1}$; MS (FAB): m/z $420(\mathrm{M}+\mathrm{H})$.<smiles>CC(=O)N(C1CC1)C(C(=O)Nc1ccccc1OC(=O)c1ccccc1)C(C)C</smiles>

2-(2-( $N$-cyclopropylacetamido)-3-methylbutanamido)phenyl benzoate (22g): Prepared according to the general procedure from $10 \mathrm{~g}$ for $15 \mathrm{~h}$ to provide title compound as a colorless sticky liquid (83\%) after purification by flash column chromatography on silica gel, eluting with hexanes to 4:1 hexanes/ethyl acetate gradient. ${ }^{1} \mathrm{H}$ NMR (300 MHz, $\mathrm{CDCl}_{3}$ ): $\delta 9.69$ (br s, 1H), 8.28-8.23 (m, 3H), 7.66 (t, $\left.J=7.5 \mathrm{~Hz}, 1 \mathrm{H}\right), 7.54$ (t, $J=7.5 \mathrm{~Hz}$, 2H), 7.28-7.22 (m, 1H), 7.12 (d, $J=3.6 \mathrm{~Hz}, 2 \mathrm{H}), 3.90$ (d, $J=11.1 \mathrm{~Hz}, 1 \mathrm{H}), 2.81-2.68$ (m, $1 \mathrm{H}), 2.66-2.59(\mathrm{~m}, 1 \mathrm{H}), 1.81(\mathrm{~s}, 3 \mathrm{H}), 1.03-0.97(\mathrm{~m}, 4 \mathrm{H}), 0.87-0.74(\mathrm{~m}, 6 \mathrm{H}) ;{ }^{13} \mathrm{C} \mathrm{NMR}$ (75 MHz, $\left.\mathrm{CDCl}_{3}\right): \delta 175.7,170.4,164.8,141.0,133.9,131.4,130.7,129.4,128.7,126.6$, 124.5, 122.7, 122.5, 72.9, 32.8, 26.5, 22.8, 20.2, 9.8, 9.0; IR (film): 3222, 2967, 2873, 1741, 1685, 1634, 1607, 1533, 1450, 1388, 1367, 1302, 1241, 1174, 1103, 1055, 1023 $\mathrm{cm}^{-1}$; MS (FAB): $\mathrm{m} / \mathrm{z} 395(\mathrm{M}+\mathrm{H})$. 
<smiles>CCC(=O)N(Cc1ccccc1)C(C(=O)OC)C(C)C</smiles>

Methyl 2-(N-benzylpropionamido)-3-methylbutanoate (15): To a solution of Ugi product $(0.3 \mathrm{mmol})$ in methanol $(2.5 \mathrm{~mL})$ was added acetyl chloride $(1.5 \mathrm{mmol})$ in one portion. The flask was equipped with a reflux condenser and heated to $55^{\circ} \mathrm{C}$ for $3 \mathrm{~h}$. The reaction was cooled to room temperature and the solvent was removed in vacuo. The resulting residue was purified by flash column chromatography on silica gel, eluting with hexanes to 19:1 hexanes/ethyl acetate gradient to provide the title compound as a colorless liquid. The title compound exists as a two rotamers in 7:3 ratio at room temperature in chloroform. ${ }^{1} \mathrm{H}$ NMR (300 MHz, $\left.\mathrm{CDCl}_{3}\right)$ : $\delta$ 7.34-7.13 (m, 5H), 4.99-4.94 (m, 1H), 4.62 (s, 1.4H), 4.26 (d, $J=15.3 \mathrm{~Hz}, 0.3 \mathrm{H}), 4.02$ (d, $J=11.1 \mathrm{~Hz}, 0.3 \mathrm{H}), 3.43$ (s, 2.1H), 3.32 (s, 0.9H), 2.61-2.21 (m, 3H), 1.24 (t, $J=7.8 \mathrm{~Hz}, 0.9 \mathrm{H}), 1.11$ (t, $J=7.8 \mathrm{~Hz}$, 2.1H), 0.97 (d, $J=6.3 \mathrm{~Hz}, 3 \mathrm{H}), 0.88$ (d, $J=6.6 \mathrm{~Hz}, 2.1 \mathrm{H}$ ), 0.83 (d, $J=6.6 \mathrm{~Hz}, 0.9 \mathrm{H}) ;{ }^{13} \mathrm{C}$ NMR (75 MHz, $\left.\mathrm{CDCl}_{3}\right): \delta 175.5,175.1,171.3,170.5,138.2,137.4,128.8,128.3,127.9$, 127.4, 126.9, 126.0, 65.9, 61.9, 51.9, 51.7, 48.4, 45.9, 28.0, 27.6, 27.1, 27.0, 20.1, 20.0, 18.9, 9.8; IR (film): 2966, 2876, 1737, 1652, 1497, 1453, 1435, 1411, 1370, 1300, 1255 , 1227, 1200, 1167, 1131, 1074, $1010 \mathrm{~cm}^{-1}$; MS (CI): m/z $278(\mathrm{M}+\mathrm{H})$.<smiles>COC(=O)C(C(C)C)N(C(C)=O)C1CC1</smiles>

Methyl 2-(N-cyclopropylacetamido)-3-methylbutanoate (23): To a solution of Ugi product $(0.06 \mathrm{mmol})$ in methanol $(0.1 \mathrm{~mL})$ was added a solution of acetyl chloride $(0.3$ mmol) in methanol $(0.5 \mathrm{~mL})$ in one portion. The resulting solution was stirred at room temperature until the starting material disappeared. The solvent was removed in vacuo and purified by flash column chromatography on silica gel, eluting with hexanes to 7:3 hexanes/ethyl acetate gradient to provide the title compound as a colorless liquid. ${ }^{1} \mathrm{H}$ NMR (300 MHz, $\mathrm{CDCl}_{3}$ ): $\delta 3.95$ (d, $\left.J=10.2 \mathrm{~Hz}, 1 \mathrm{H}\right), 3.67$ (s, 3H), 2.76-2.69 (m, 1H), 2.65-2.52 (m, 1H), 2.23 (s, 3H), 1.08 (d, $J=6.6 \mathrm{~Hz}, 3 \mathrm{H}), 0.91-0.88$ (m, 4H), 0.85 (d, $J=$ 
$6.6 \mathrm{~Hz}, 3 \mathrm{H}) ;{ }^{13} \mathrm{C}$ NMR $\left(75 \mathrm{MHz}, \mathrm{CDCl}_{3}\right): \delta 173.9,172.0,66.8,52.1,32.2,27.9,22.9$, 21.8, 20.1, 10.2, 9.4; IR (film): 2965, 2874, 1740, 1656, 1434, 1411, 1383, 1366, 1300, 1266, 1202, 1131, $1015 \mathrm{~cm}^{-1}$; MS (CI): m/z 214 (M + H).<smiles>CCC(=O)OC(C(=O)Nc1ccccc1OC(=O)CC(C)C)C(C)C</smiles>

24: To a solution of $10 \mathrm{~b}(0.13 \mathrm{~g}, 0.64 \mathrm{mmol})$ in $\mathrm{CH}_{2} \mathrm{Cl}_{2}(1.5 \mathrm{~mL})$ were added isobutyraldehyde $(0.058 \mathrm{~mL}, 0.64 \mathrm{mmol})$ followed by propionic acid $(0.048 \mathrm{~mL}, 0.64$ $\mathrm{mmol}$ ), and the reaction mixture was stirred at room temperature for $18 \mathrm{~h}$. After completion of the reaction, solvent was removed in vacuo, and the residue was purified by flash column chromatography on silica gel, eluting with hexanes to 10:1 hexanes/ethyl acetate gradient afforded the title compound $24(0.154 \mathrm{~g}, 69 \%)$ as a reddish orange liquid. ${ }^{1} \mathrm{H}$ NMR (300 MHz, $\mathrm{CDCl}_{3}$ ): $\delta 8.13$ (d, $J=8.1 \mathrm{~Hz}, 1 \mathrm{H}$ ), 7.82 (s, $1 \mathrm{H}$ ), 7.24 (td, $J=8.1$, $1.8 \mathrm{~Hz}, 1 \mathrm{H}$ ), 7.17 (dd, $J=8.4,1.8 \mathrm{~Hz}, 1 \mathrm{H}$ ), 7.09 (td, $J=8.4,1.8 \mathrm{~Hz}, 1 \mathrm{H}$ ), 5.27 (d, $J=4.5$ Hz, 1H), 2.48 (q, $J=7.8 \mathrm{~Hz}, 2 \mathrm{H}), 2.38-2.35$ (m, 1H), 1.41 (s, 9H), $1.22(\mathrm{t}, J=7.8 \mathrm{~Hz}$, 3H), 0.99 (d, $J=7.2 \mathrm{~Hz}, 3 \mathrm{H}), 0.98$ (d, $J=7.2 \mathrm{~Hz}, 3 \mathrm{H}) ;{ }^{13} \mathrm{C} \mathrm{NMR}\left(75 \mathrm{MHz}, \mathrm{CDCl}_{3}\right): \delta$ 176.5, 173.2, 167.9, 141.5, 129.3, 126.5, 125.5, 123.5, 122.1, 78.2, 39.5, 31.1, 27.7, 27.4, 19.0, 17.2, 9.3; IR (film): 3428, 2970, 2877, 1752, 1697, 1608, 1521, 1453, 1177, 1159, 1105, $1026 \mathrm{~cm}^{-1}$; MS (CI): m/z $367\left(\mathrm{M}+\mathrm{NH}_{4}\right), 350(\mathrm{M}+\mathrm{H})$.

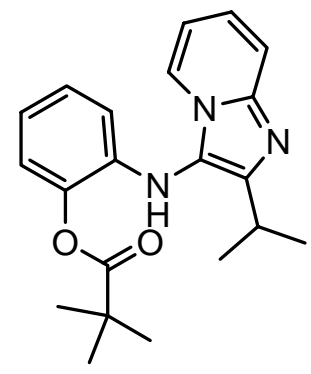

2-(2-isopropylH-imidazo[1,2-a]pyridine-3-ylamino)phenyl pivalate (25): 2-Amino pyridine $(0.135 \mathrm{~g}, 1.43 \mathrm{mmol})$ was dissolved in methanol $(3 \mathrm{~mL})$. Isobutyraldehyde $(0.2$ $\mathrm{mL}, 2.19 \mathrm{mmol})$ and $\mathbf{1 0 b}(0.35 \mathrm{~g}, 1.72 \mathrm{mmol})$ were added at room temperature. A $1 \mathrm{M}$ solution of perchloric acid in methanol $(0.15 \mathrm{~mL})$ was added, and stirred at the same 
temperature for $18 \mathrm{~h}$. The reaction mixture was diluted with dichloromethane (30 mL) and extracted successively with water $(30 \mathrm{~mL})$, a saturated aqueous solution of $\mathrm{NaHCO}_{3}$ $(15 \mathrm{~mL})$, and brine $(30 \mathrm{~mL})$. After that the organic layer was dried and concentrated in vacuo to give yellowish solid, which was purified by flash column chromatography on silica gel, eluting with hexanes to 3:2 hexanes/ethyl acetate gradient afforded the title compound 25 (0.39 g, 78\%) as a ivory white solid. mp: 158.0-159.2 ${ }^{\circ} \mathrm{C} ;{ }^{1} \mathrm{H}$ NMR (300 $\mathrm{MHz}, \mathrm{CDCl}_{3}$ ): $\delta 7.78$ (dd, $J=6.9,0.9 \mathrm{~Hz}, 1 \mathrm{H}$ ), 7.59 (dd, $J=9.0,1.2 \mathrm{~Hz}, 1 \mathrm{H}$ ), 7.16 (ddd, $J=8.7,7.2,1.2 \mathrm{~Hz}, 1 \mathrm{H}), 7.05$ (d, $J=7.8 \mathrm{~Hz}, 1 \mathrm{H}), 6.94$ (t, $J=7.8 \mathrm{~Hz}, 1 \mathrm{H}), 6.81$ (td, $J=$ 7.8, $1.2 \mathrm{~Hz}, 1 \mathrm{H}$ ), 6.72 (t, $J=7.2 \mathrm{~Hz}, 1 \mathrm{H}), 6.17$ (d, $J=7.8 \mathrm{~Hz}, 1 \mathrm{H}), 5.29$ (s, $1 \mathrm{H}), 3.14$ (sep, $J=6.9 \mathrm{~Hz}, 1 \mathrm{H}), 1.44$ (s, 9H), 1.32 (d, $J=6.9 \mathrm{~Hz}, 6 \mathrm{H}) ;{ }^{13} \mathrm{C} \mathrm{NMR}\left(75 \mathrm{MHz}, \mathrm{CDCl}_{3}\right)$ : $\delta$ 176.8, 148.6, 142.9, 138.4, 137.6, 127.0, 124.4, 122.7, 122.6, 119.7, 117.6, 116.2, 113.6, 112.1, 39.7, 27.5, 26.9, 22.5; IR (film): 3254, 2966, 2934, 1756, 1632, 1607, 1573, 1501, 1396, 1340, 1272, 1249, 1173, $1096 \mathrm{~cm}^{-1}$; MS (CI): m/z 352 (M + H). 